\title{
Effect of astragaloside IV against rat myocardial cell apoptosis induced by oxidative stress via mitochondrial ATP-sensitive potassium channels
}

\author{
FENG-YING GUAN ${ }^{1}$, SHI-JIE YANG ${ }^{1}$, JINXIANG LIU $^{2}$ and SI-RUI YANG ${ }^{2}$ \\ ${ }^{1}$ Department of Pharmacology, College of Basic Medical Sciences, Jilin University; ${ }^{2}$ Department of Pediatric Cardiology, \\ Institute of Pediatrics, The First Affiliated Hospital of Jilin University, Changchun, Jilin 130021, P.R. China
}

Received May 9, 2014; Accepted February 13, 2015

DOI: $10.3892 / \mathrm{mmr} .2015 .3400$

\begin{abstract}
Astragaloside is one of the most common traditional Chinese medicines and is derived from Astragalus membranaceus. Astragaloside IV (AsIV) is a monomer located in an extract of astragaloside. The current study investigated the protective effects of AsIV against hydrogen peroxide $\left(\mathrm{H}_{2} \mathrm{O}_{2}\right)$-induced injury in cardiocytes and elucidated the mechanisms responsible for this protective effect. Cultured neonatal rat cardiocytes were divided into five experimental groups as follows: i) Dimethyl sulfoxide; ii) $\mathrm{H}_{2} \mathrm{O}_{2}$; iii) AsIV $+\mathrm{H}_{2} \mathrm{O}_{2}$; iv) AsIV $+\mathrm{H}_{2} \mathrm{O}_{2}+5$-hydroxydecan oate (5-HD); and v) nicorandil $+\mathrm{H}_{2} \mathrm{O}_{2}$. Cardiocyte survival was analyzed using an MTT assay. Lactate dehydrogenase (LDH) release was also assessed to evaluate the viability of the cells. Intracellular reactive oxygen species (ROS) were measured by 2,7-dichlorodihydrofluorescein diacetate staining. The apoptotic rate was measured by flow cytometry. Mitochondrial membrane potential $(\Delta \Psi \mathrm{m})$ and intracellular calcium were observed using a laser confocal microscopy system. The results indicated that AsIV promoted the survival of cardiocytes $(\mathrm{P}<0.05)$, attenuated $\mathrm{LDH}$ release $(\mathrm{P}<0.05)$, ROS production $(\mathrm{P}<0.01)$ and apoptosis $(\mathrm{P}<0.01)$, stabilized the $\Delta \Psi \mathrm{m}$ and reduced intracellular calcium overload $(\mathrm{P}<0.01)$ compared with the $\mathrm{H}_{2} \mathrm{O}_{2}$ group. The mitochondrial adenosine triphosphate-sensitive potassium channel $\left(\right.$ mitoK $\left._{\mathrm{ATP}}\right)$ inhibitor 5-HD was observed to partially reverse the protective effect of AsIV. Following treatment with 5-HD, the survival of cardiocytes was reduced $(\mathrm{P}<0.05), \mathrm{LDH}$ release $(\mathrm{P}<0.01)$ and ROS production $(\mathrm{P}<0.05)$ were stimulated, $\Delta \Psi \mathrm{m}$ and intracellular calcium change were increased $(\mathrm{P}<0.01)$ and apoptosis
\end{abstract}

Correspondence to: Dr Si-Rui Yang, Department of Pediatric Cardiology, Institute of Pediatrics, The First Affiliated Hospital of Jilin University, 71 Xinmin Street, Changchun, Jilin 130021, P.R. China

E-mail: siruiyang@163.com

Key words: astragaloside IV, mitochondrial membrane potential, intracellular calcium, reactive oxygen species, apoptosis, mitochondrial adenosine triphosphate-sensitive potassium channels was increased $(\mathrm{P}<0.01)$ compared with the AsIV+ $\mathrm{H}_{2} \mathrm{O}_{2}$ group. Thus, AsIV has potential for use in the suppression of apoptosis resulting from $\mathrm{H}_{2} \mathrm{O}_{2}$ exposure, and mitoK $\mathrm{K}_{\text {ATP }}$ activation may underlie this protective mechanism.

\section{Introduction}

Reperfusion is the most effective method of limiting acute myocardial ischemia necrosis. However, reperfusion may also be associated with a burst of reactive oxygen species (ROS) production and intracellular calcium overload (1-3). These dichotic effects result in paradoxical cardiocyte dysfunction, a phenomenon termed myocardial ischemia reperfusion injury (MIRI). The role of mitochondria during MIRI is critical, as apoptosis is promoted by the mitochondrial pathway associated with the mitochondrial permeability transition (MPT). MPT pores (MPTPs) are located in the inner mitochondrial membrane, and when MIRI induces cellular dysfunction, including increased $\mathrm{Ca}^{2+}$ concentrations and oxidative stress, mitochondria undergo swelling and become uncoupled due to the opening of MPTPs (4). This leads to matrix swelling, release of apoptotic signaling molecules and irreversible injury to the mitochondria (5). The role of the mitoK $\mathrm{ATP}_{\mathrm{A}}$ channel in modulating cardiac mitochondrial function has been investigated previously $(6,7)$. Wakiyama et al (8) confirmed that the opening of mitochondrial adenosine triphosphate-sensitive potassium channels $\left(\right.$ mitoK $_{\mathrm{ATPs}}$ ) may reduce the release of cytochrome $c$, inhibit caspase- 3 activation, stabilize the mitochondrial membrane potential (MMP) and inhibit apoptosis (8).

Astragaloside is one of the most common Traditional Chinese Medicines. It possesses multiple physiological and pharmacological functions, including a protective effect in the myocardium following ischemic injury. This effect may be associated with clearing oxygen free radicals or reducing blood viscosity $(9,10)$. Notably, prior work has demonstrated that Astragalus injection (Huangqizhusheye) may activate the mitoK $_{\text {ATPs }}$ and reduce MIRI (11). Astragaloside IV (AsIV) is an extract of the monomer astragaloside (Fig. 1). AsIV produces various effects, including protection against cerebral ischemia-reperfusion injury, and the attenuation of renal tubulointerstitial fibrosis and diabetes (12-14). Certain 
protective effects of AsIV on cardiovascular disease have also been suggested (15-19). In addition to providing cardioprotection during myocardial ischemia $(18,19)$, AsIV has also been demonstrated to limit endothelial dysfunction induced by oxidative stress (15) and inhibit compensatory hypertrophy of myocardial cells (16). The results obtained in these previous studies provided the impetus to study the mechanism of AsIV protection during reperfusion.

In the current study, the protective role of mitoK $\mathrm{K}_{\mathrm{ATP}}$ following AsIV treatment was investigated in cardiocytes. Oxidative stress in cardiocytes was stimulated by treating cultured cells with $0.2 \mathrm{mmol} / \mathrm{l}$ hydrogen peroxide $\left(\mathrm{H}_{2} \mathrm{O}_{2}\right)(20)$, resulting in calcium overload and damage to the MMP. These three factors may act separately, or interact with each other to cause damage to cellular structure, function and metabolism, and ultimately result in cell death. This type of injury simulates the pathogenesis of MIRI; thus, the present study used this model to analyze the effect of AsIV on MIRI and its underlying mechanisms.

\section{Materials and methods}

Cell culture and reagents. Primary rat neonatal cardiocytes were derived from 1-2-day-old Wistar rats (Experimental Animal Holding Facility of Jilin University, China). Briefly, the hearts were removed under aseptic conditions and the ventricles were homogenized in D-Hank's buffer. The tissue fragments were digested by stepwise exposure to $0.125 \%$ pancreatin (Gibco Life Technologies, Carlsbad, CA, USA). The dissociated cells were pre-plated for $90 \mathrm{~min}$ to remove fibroblasts, and the non-adherent cardiocytes were then plated at a density of $1 \times 10^{5}$ cells $/ \mathrm{mm}^{2}$. Cells were cultured in Iscove's modified Dulbecco's medium supplemented with $10 \%$ fetal bovine serum and $1 \%$ penicillin/streptomycin (BHKT Clinical Reagent Co., Ltd, Beijing, China), and maintained at $37^{\circ} \mathrm{C}$ in an incubator with $95 \% \mathrm{O}_{2}$ and $5 \% \mathrm{CO}_{2}$. All animal studies were performed in accordance with the Guide for the Care and Use of Laboratory Animals and approved by the Institutional Animal Care and Use Committee of Jilin University (Changchun, China).

2,7-Dichlorofluorescein diacetate (DCFH), Fluo-3/AM, Rhodamine 123 and Annexin V were obtained from Sigma-Aldrich (St. Louis, MO, USA). The mitoK ${ }_{\text {ATP }}$ inhibitor 5-hydroxydecanoate (5-HD) was also obtained from Sigma-Aldrich.

Peroxidation injury. To induce oxidative stress, cells were cultured in serum-free medium for $12 \mathrm{~h}$ when the cells were semiconfluent and beating synchronously. For the peroxidation challenge, the cells were cultured in medium with $0.2 \mathrm{mmol} / 1 \mathrm{H}_{2} \mathrm{O}_{2}$ (Beijing Chemical Factory, Beijing, China) and maintained at $37^{\circ} \mathrm{C}$ for $24 \mathrm{~h}$.

Experimental design. The present study utilized five experimental groups of cardiocytes as follows: i) The dimethyl sulfoxide (DMSO, Beijing Chemical Factory, Beijing, China) group, maintained in $0.1 \%$ DMSO; ii) the $\mathrm{H}_{2} \mathrm{O}_{2}$ group, treated with $0.2 \mathrm{mmol} / 1 \mathrm{H}_{2} \mathrm{O}_{2}$ for $24 \mathrm{~h}$; iii) the AsIV $+\mathrm{H}_{2} \mathrm{O}_{2}$ group, pretreated with $30 \mathrm{mg} / \mathrm{l}$ AsIV (provided by Academy of Chinese Medical Sciences of Jilin Province, Changchun, China)

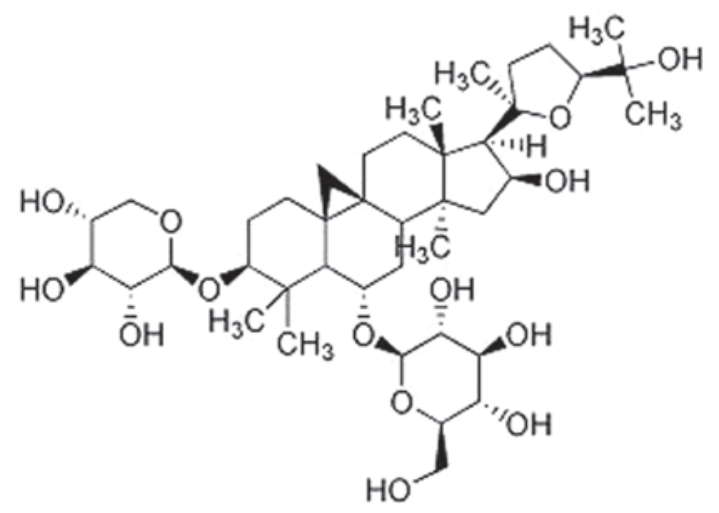

Figure 1. Chemical structure of astragaloside IV.

for 30 min prior to $\mathrm{H}_{2} \mathrm{O}_{2}$ injury; iv) the AsIV+ $\mathrm{H}_{2} \mathrm{O}_{2}+5-\mathrm{HD}$ group, pretreated with $50 \mu \mathrm{mol} / 15$-HD for $5 \mathrm{~min}$ prior to the AsIV pretreatment; and v) the nicorandil (NIC; Changchun Dazheng International Trade Group Pharmaceutical Co., Ltd, Changchun, China) $+\mathrm{H}_{2} \mathrm{O}_{2}$ group, pretreated with $120 \mathrm{mg} / \mathrm{l}$ NIC for 30 min prior to $\mathrm{H}_{2} \mathrm{O}_{2}$ injury. The $\mathrm{NIC}+\mathrm{H}_{2} \mathrm{O}_{2}$ group was used as a positive control. For all AsIV treatments, a $0.1 \%$ AsIV stock solution was dissolved in DMSO.

Cardiocyte viability and lactate dehydrogenase ( $L D H)$ activity. Cardiocyte survival was assessed using an MTT assay. Briefly, cells were incubated with $200 \mu 1$ medium in 96-well plates, $5 \mu 1$ MTT stock solution $(5 \mathrm{mg} / \mathrm{ml})$ was then added to each well and the cells were incubated for a further $4 \mathrm{~h}$. Blue formazan precipitate was produced from the cells by adding $100 \mu \mathrm{l}$ DMSO and gently shaking, and the absorbance was measured at $570 \mathrm{~nm}$ using an A-5082 ELISA reader (Chengdu Taimeng Technology Co., Ltd., Chengdu, China). LDH release was measured using a GF-200 Semi Automatic Biochemical Analyzer (Shandong Gaomi Caihong Instruments Co., Ltd., Gaomi, China).

Analysis of ROS production. Intracellular ROS levels were analyzed using a flow cytometer with DCFH staining. DCFH was dissolved in ethanol (Beijing Chemical Factory, Beijing, China) to produce a $1 \mathrm{mmol} / 1$ stock solution. Cells were subsequently washed three times with phosphate-buffered saline (PBS) prior to the addition of DCFH $(10 \mu \mathrm{M})$. Following a 10 -min incubation in the dark, the cells were washed three times then resuspended in PBS. Fluorescence was measured using a fluorescence microplate reader at 488/525 nm (Gemini XPS; Shanghai Spectrum Instruments Co., Ltd., Shanghai, China).

Apoptosis analysis. The non-adherent myocytes were digested by treatment with $0.25 \%$ pancreatin for $10 \mathrm{~min}$. The cells were then washed twice with PBS, and the percentage of apoptotic cells was determined with an Coulter Epics XL flow cytometer (Beckman Coulter, Brea, CA, USA) after double staining with the Annexin V-FITC apoptosis detection kit (Tianjin Sungene Biotech Co., Ltd. China). Annexin V was used as an apoptosis indicator and propidium iodide (PI) as a necrosis indicator. $\mathrm{An}^{+} \mathrm{PI} \mathrm{I}^{-}$represents viable apoptotic cells in the lower right quadrant. 


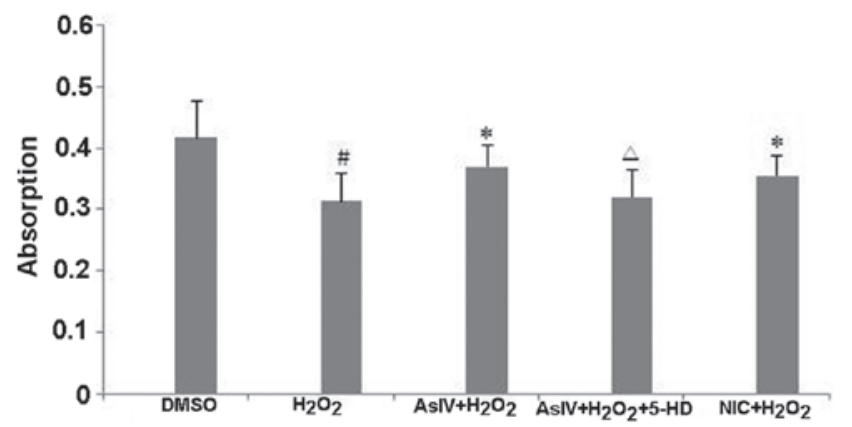

Figure 2. Viability of primary cultured neonatal rat myocardial cells (MTT. $\left.\mathrm{A}_{490}\right)$. ${ }^{\prime \prime} \mathrm{P}<0.05$ vs. the DMSO group, ${ }^{\prime} \mathrm{P}<0.05$ vs. the $\mathrm{H}_{2} \mathrm{O}_{2}$ group, ${ }^{\Delta} \mathrm{P}<0.05$ vs. the AsIV $+\mathrm{H}_{2} \mathrm{O}_{2}$ group. DMSO, dimethyl sulfoxide; $\mathrm{H}_{2} \mathrm{O}_{2}$, hydrogen peroxide; AsIV, astragaloside IV; 5-HD, 5-hydroxydecanoate; NIC, nicorandil.

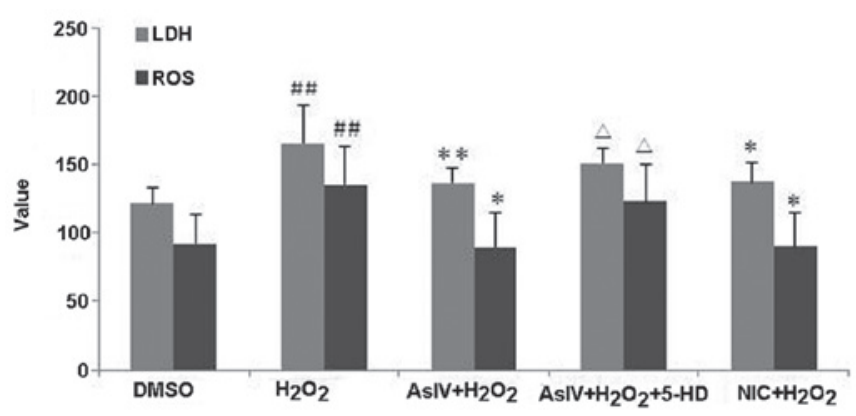

Figure 3. LDH and ROS levels in rat myocardial cells. (LDH, U/1; ROS, optical density $\left.{ }_{525} / \mathrm{a} . u\right) .{ }^{\#} \mathrm{P}<0.01$ vs. the DMSO group, ${ }^{* *} \mathrm{P}<0.01$ vs. the $\mathrm{H}_{2} \mathrm{O}_{2}$ group, ${ }^{\Delta} \mathrm{P}<0.05$ vs. the AsIV+ $\mathrm{H}_{2} \mathrm{O}_{2}$ group. $\mathrm{LDH}$, lactate dehydrogenase; $\mathrm{ROS}$, reactive oxygen species; DMSO, dimethyl sulfoxide; $\mathrm{H}_{2} \mathrm{O}_{2}$, hydrogen peroxide; AsIV, astragaloside IV; 5-HD, 5-hydroxydecanoate; NIC, nicorandil.

Cytosolic calcium concentration, $\left[\mathrm{Ca}^{2+}\right] i$. Fluo- 3 was used as $\mathrm{a} \mathrm{Ca}^{2+}$ indicator during fluorescence imaging to assess $\left[\mathrm{Ca}^{2+}\right] \mathrm{i}$. Changes in $\left[\mathrm{Ca}^{2+}\right]$ i were analyzed using a Fluoview FV500 confocal laser scanning microscope (Olympus Corporation, Tokyo, Japan). Cells in 24-well plates were washed with HEPES buffer and treated with Fluo-3/AM $(10 \mu \mathrm{mol} / \mathrm{l})$ for $40 \mathrm{~min}$ in the dark. Following removal of Fluo-3/AM, the cells were washed three times with HEPES, and fresh HEPES was added with the various drug treatments as described. Baseline fluorescence in normal cells was recorded for $1 \mathrm{~min}$, then $\mathrm{H}_{2} \mathrm{O}_{2}$ was added and the change in fluorescence was measured for $5 \mathrm{~min}$. The results were analyzed using software which accompanied the confocal microscope. Alterations in the levels of green fluorescence were used to evaluate $\left[\mathrm{Ca}^{2+}\right] \mathrm{i}$.

$M M P(\Delta \Psi m)$. Fluorescence imaging of $\Delta \Psi \mathrm{m}$ was conducted using Rhodamine 123 as an indicator. Rhodamine 123 is a mitochondria-selective dye, which in a reaction driven by $\Delta \Psi \mathrm{m}$ in normal polarized mitochondria, assembles into red fluorescence-emitting dimers forming J-aggregates. Changes in MMP were analyzed using the confocal microscope. Cells in 24-well plates were treated with Rhodamine 123 $(5 \mu \mathrm{g} / \mathrm{ml})$ for 10 min away from light. Following removal of Rhodamine 123, the cells were washed with PBS. The level of Rhodamine 123 was measured and analyzed with the Fluoview Viewer software, version 1.7a (Olympus Corporation).
Statistics. Data are expressed as the mean \pm standard error of the mean. Statistical significance was calculated using one-way analysis of variance with Newman-Keuls post hoc test. $\mathrm{P}<0.05$ was considered to indicate a statistically significant difference.

\section{Results}

Effect of AsIV on cardiocyte viability and the change in LDH activity. Pretreatment with AsIV significantly increased cardiocyte viability compared with that of the $\mathrm{H}_{2} \mathrm{O}_{2}$ group $(\mathrm{P}<0.05)$, while pretreatment with 5-HD resulted in reduced viability ( $\mathrm{P}<0.05$ vs. the AsIV group) (Fig. 2). As indicated in Fig. 3, cardiocyte damage resulting from $\mathrm{H}_{2} \mathrm{O}_{2}$ exposure was reflected by increased LDH release $(\mathrm{P}<0.01$ vs. the DMSO control group); which was reduced by AsIV pretreatment ( $\mathrm{P}<0.01$ vs. the $\mathrm{H}_{2} \mathrm{O}_{2}$ group) and significantly increased in the 5 -HD pretreatment group ( $\mathrm{P}<0.05$ vs. the AsIV group). These results indicated that AsIV may protect cardiocytes against $\mathrm{H}_{2} \mathrm{O}_{2}$ injury, while the mitoK $\mathrm{K}_{\text {ATP }}$ inhibitor 5-HD may partially reverse this effect.

AsIV alters oxidative stress and $\left[\mathrm{Ca}^{2+}\right] \mathrm{i}$. Elevated levels of ROS may contribute to ischemia-reperfusion injury. Therefore, the present study evaluated whether AsIV-mediated cardioprotection may be partially attributed to a reduction in oxidative stress. ROS generation was assessed by DCFH fluorescence. The results indicated that $\mathrm{H}_{2} \mathrm{O}_{2}$ induced significant ROS generation in cardiocytes compared with the DMSO control group $(\mathrm{P}<0.01)$ (Fig. 3), which was reduced following AsIV treatment $\left(\mathrm{P}<0.05\right.$ vs. the $\mathrm{H}_{2} \mathrm{O}_{2}$ group). This protective effect was significantly attenuated by 5 -HD pretreatment $(\mathrm{P}<0.05)$. These results indicate that AsIV may protect cardiocytes against $\mathrm{H}_{2} \mathrm{O}_{2}$ injury by reducing oxidative stress; and this effect may be associated with the mitoK $_{\text {ATP }}$.

$\mathrm{Ca}^{2+}$ overload is a key contributor to the mitochondrial permeability transition leading to ischemia-reperfusion injury. As displayed in Fig. 4, AsIV-pretreated cells presented significantly lower $\left[\mathrm{Ca}^{2+}\right] \mathrm{i}$ compared with that of the $\mathrm{H}_{2} \mathrm{O}_{2}$ group $(\mathrm{P}<0.01)$. 5-HD pretreatment prior to AsIV exposure resulted in significantly higher $\left[\mathrm{Ca}^{2+}\right] \mathrm{i}$ compared with that of the AsIV group $(\mathrm{P}<0.01)$. These results indicated that AsIV may relieve $\mathrm{Ca}^{2+}$ overload, an effect which is also associated with the mitoK $_{\text {ATP. }}$.

Changes in MMP. MPTP opening may be critical for the transition from reversible to irreversible myocardial ischemia-reperfusion injury. Therefore, the present study investigated MPTP dynamics in cardiocytes using Rhodamine 123. The MMP was $(93.80 \pm 19.53) 30 \mathrm{~min}$ subsequent to AsIV treatment, which was significantly higher than that in the $\mathrm{H}_{2} \mathrm{O}_{2}$ group $(\mathrm{P}<0.01)$ (Fig. 5). 5-HD pretreatment prior to AsIV led to an MMP that was significantly lower than that of the AsIV group $(\mathrm{P}<0.01)$. These results demonstrated that AsIV may protect cardiocytes by stabilizing the MMP, and this effect may be associated with the mitoK $\mathrm{ATP}_{\text {. }}$.

Changes in the rate of apoptosis. The effect of AsIV on cell apoptosis was analyzed by measuring Annexin $\mathrm{V}$ positivity using flow cytometry. Pretreatment with AsIV led to significantly reduced Annexin V-positivity compared with the $\mathrm{H}_{2} \mathrm{O}_{2}$ 
A

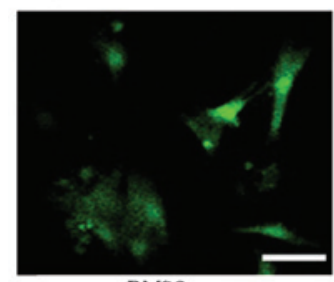

DMSO

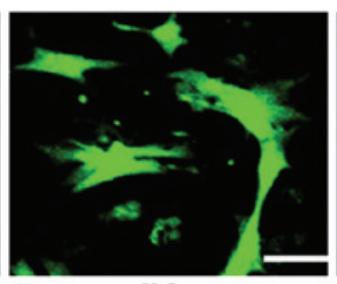

$\mathrm{H}_{2} \mathrm{O}_{2}$

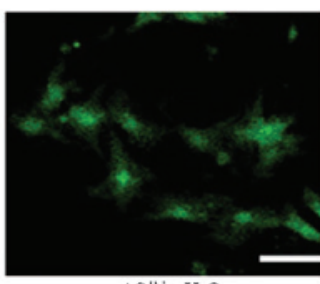

$\mathrm{ASI}+\mathrm{H}_{2} \mathrm{O}$

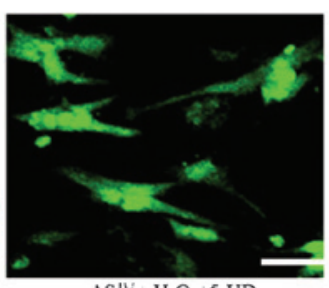

ASIV $-\mathrm{H}_{2} \mathrm{O}_{2}+5$ - $\mathrm{HD}$

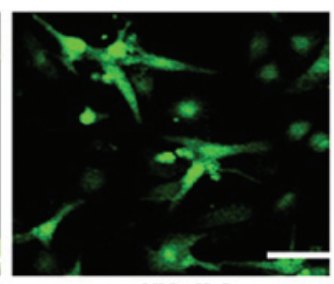

$\mathrm{NIC}+\mathrm{H}_{2} \mathrm{O}_{2}$

B

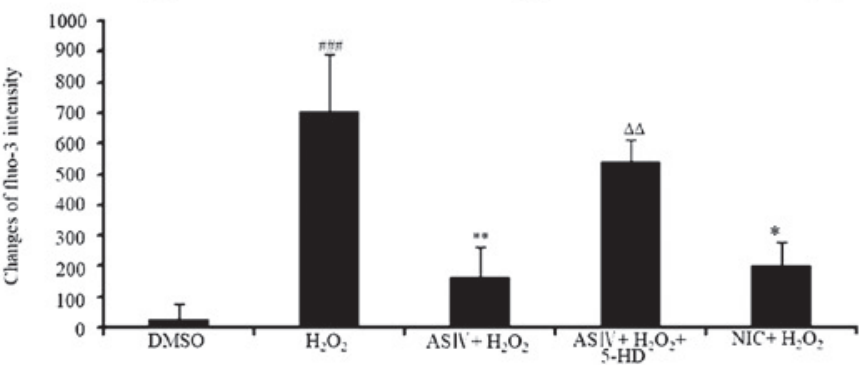

Figure 4. Effect of AsIV on the change of fluorescence intensity of cardiocytes damaged by $\mathrm{H}_{2} \mathrm{O}_{2}$. (A) Fluo-3 was used as a Ca ${ }^{2+}$ indicator during fluorescence imaging for $\left[\mathrm{Ca}^{2+}\right] \mathrm{i}$ (magnification, $\mathrm{x} 200$ ). Scale bar $=50 \mu \mathrm{m}$. (B) Quantification of the change of fluorescence intensity of Fluo-3 (n=3). ${ }^{\# \# \#<0.001 ~ v s . ~ t h e ~}$ DMSO group, ${ }^{*} \mathrm{P}<0.05$ and ${ }^{* *} \mathrm{P}<0.01$ vs. the $\mathrm{H}_{2} \mathrm{O}_{2}$ group, ${ }^{\Delta \Delta} \mathrm{P}<0.01$ vs. the AsIV $+\mathrm{H}_{2} \mathrm{O}_{2}$ group. AsIV, astragaloside IV; $\mathrm{H}_{2} \mathrm{O}_{2}$, hydrogen peroxide; DMSO, dimethyl sulfoxide; 5-HD, 5-hydroxydecanoate; NIC, nicorandil.

A

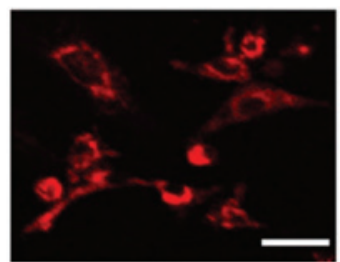

DMSO

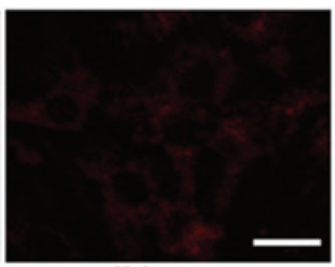

$\mathrm{H}_{2} \mathrm{O}_{2}$

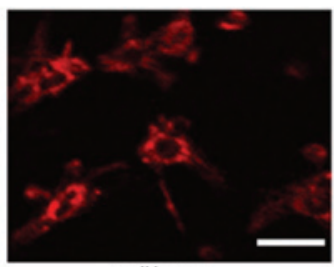

$\mathrm{ASIV}+\mathrm{H}_{2} \mathrm{O}_{2}$

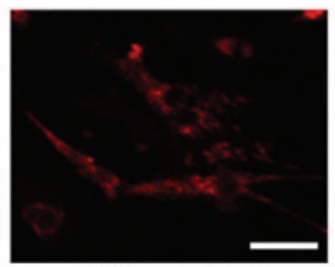

$\mathrm{ASIN}+\mathrm{H}_{2} \mathrm{O}_{2}+5-\mathrm{HD}$

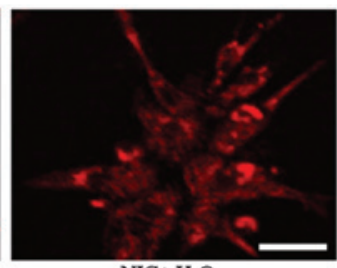

$\mathrm{NIC}+\mathrm{H}_{2} \mathrm{O}_{2}$

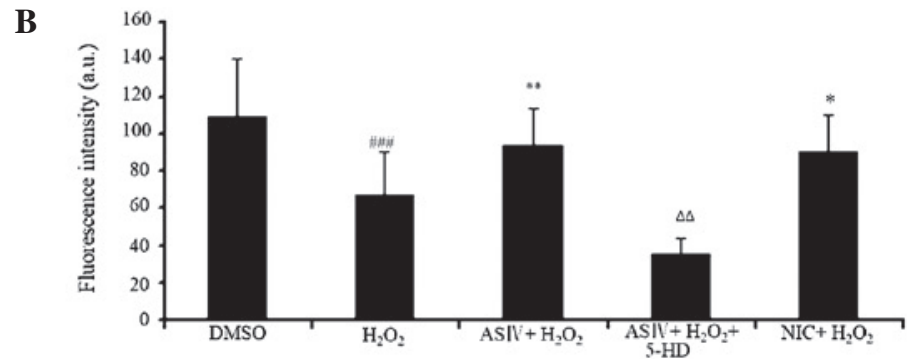

Figure 5. Effect of AsIV on $\Delta \Psi \mathrm{m}$ of cardiocytes damaged by $\mathrm{H}_{2} \mathrm{O}_{2}$. (A) Fluorescence imaging of $\Delta \Psi \mathrm{m}$ was conducted using Rhodamine 123 as a $\Delta \Psi \mathrm{m}$ indicator (magnification, x200). (B) Quantification of fluorescence intensity of Rhodamine $123(\mathrm{n}=3) .{ }^{\# \#} \mathrm{P}<0.001$ vs. the $\mathrm{DMSO}$ group, ${ }^{*} \mathrm{P}<0.05$ and ${ }^{* * *} \mathrm{P}<0.01$ vs. the $\mathrm{H}_{2} \mathrm{O}_{2}$ group, ${ }^{\Delta \Delta} \mathrm{P}<0.01$ vs. the AsIV $+\mathrm{H}_{2} \mathrm{O}_{2}$ group. $\Delta \Psi \mathrm{m}$, mitochondrial membrane potential; AsIV, astragaloside IV; $\mathrm{H}_{2} \mathrm{O}_{2}$, hydrogen peroxide; DMSO, dimethyl sulfoxide; 5-HD, 5-hydroxydecanoate; NIC, nicorandil.

group $(\mathrm{P}<0.01)$. By contrast, pretreatment with 5-HD resulted in a significant increase in the number of Annexin V-positive cells compared with the AsIV group $(\mathrm{P}<0.01)$ (Fig. 6). These results demonstrated that AsIV treatment may reduce apoptosis, and this may be associated with the mitoK $_{\text {ATP. }}$.

\section{Discussion}

The generation of ROS leads to damage to the cellular membrane, and is able to evoke calcium overload by raising membrane permeability, resulting in an inflow of calcium ions. Calcium overload may activate calcium-dependent proteinase, which catalyzes the conversion of xanthine dehydrogenase to xanthine oxidase (XO). XO promotes xanthine decomposition into uric acid with concomitant excess oxygen free radical generation. Mitochondrial $\mathrm{Ca}^{2+}$ overload, coupled with a high intracytoplasmic ROS burden, promotes the opening of the MPTPs, which induces the release of calcium from mitochondria resulting in mitochondrial swelling or failure. The combination of increased $\mathrm{ROS}, \mathrm{Ca}^{2+}$ overload and mitochondrial injury is a notable cause of cardiocyte apoptosis.

The results of the current study demonstrated that AsIV increased cardiocyte viability and reduced LDH release. Additionally, pretreatment with AsIV significantly reduced apoptosis, which demonstrated that AsIV was able to protect cardiocytes from $\mathrm{H}_{2} \mathrm{O}_{2}$ injury and inhibit apoptosis.

AsIV-mediated attenuation of ROS generation and $\mathrm{Ca}^{2+}$ overload in $\mathrm{H}_{2} \mathrm{O}_{2}$-damaged cardiocytes demonstrated that 
A

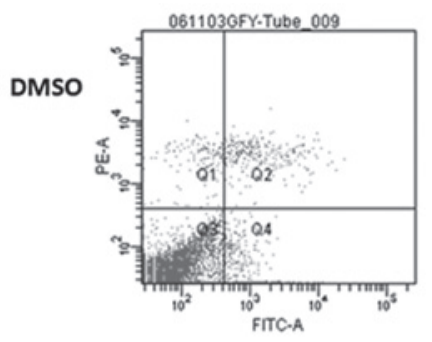

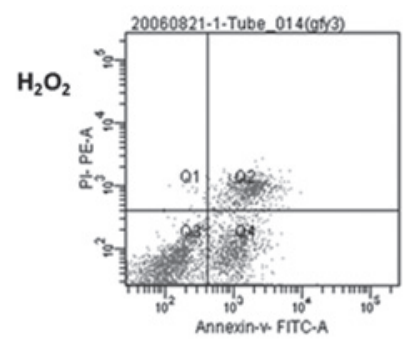
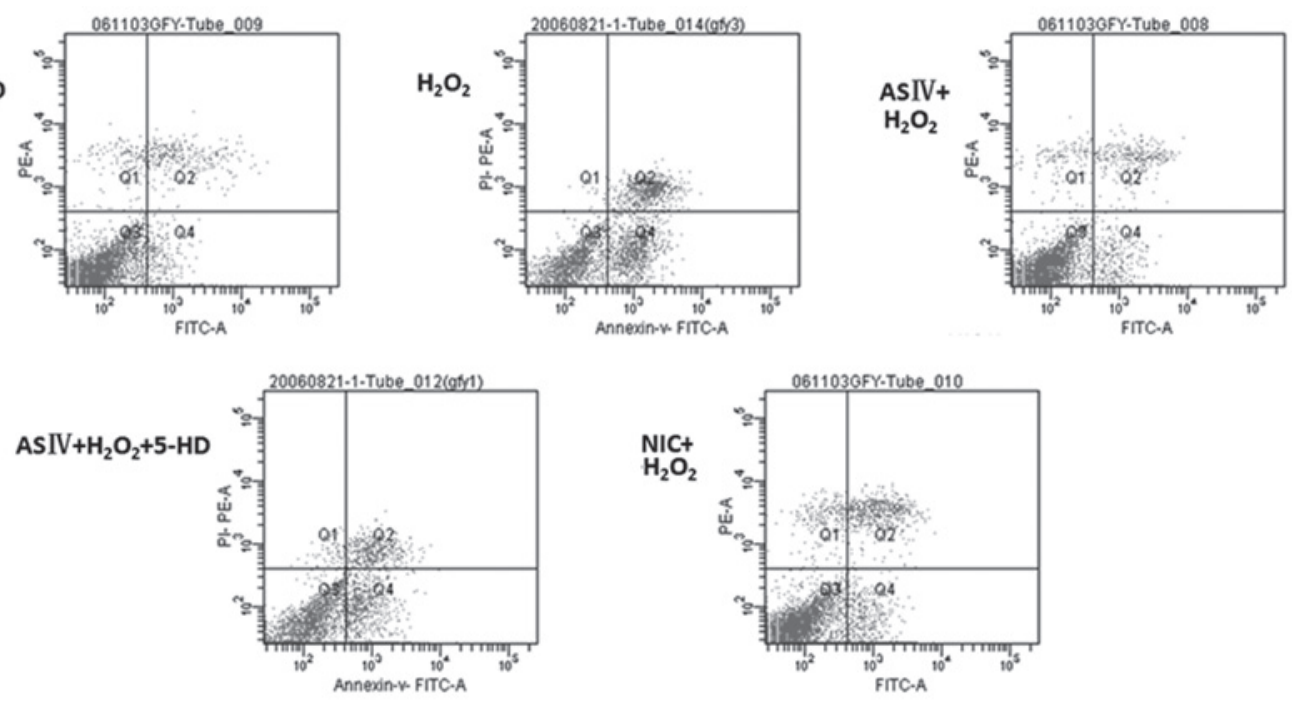

B

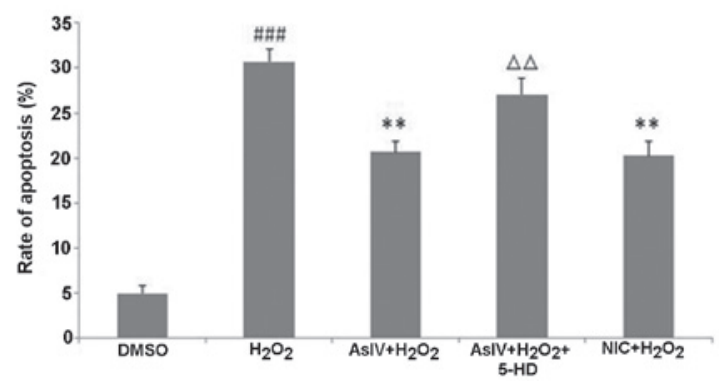

Figure 6. Effect of AsIV on apoptosis of cardiocytes damaged by $\mathrm{H}_{2} \mathrm{O}_{2}$. (A) Annexin $\mathrm{V}$ was used as an apoptosis indicator and PI as necrosis indicator. An ${ }^{+} \mathrm{PI}$ represents viable apoptotic cells in the lower right quadrant. (B) Quantification of apoptotic staining ( $\mathrm{n}=3$ ). ${ }^{\# \# \#} \mathrm{P}<$ ?? vs. the $\mathrm{DMSO}$ group, ${ }^{* *} \mathrm{P}<0.01$ vs. the $\mathrm{H}_{2} \mathrm{O}_{2}$ group, ${ }^{\Delta \Delta} \mathrm{P}<0.01$ vs. the AsIV $+\mathrm{H}_{2} \mathrm{O}_{2}$ group. AsIV, astragaloside IV; $\mathrm{H}_{2} \mathrm{O}_{2}$, hydrogen peroxide; PI, propidium iodide; An, annexin V; DMSO, dimethyl sulfoxide; FITC, fluorescein isothiocyanate.

the protective effects of AsIV are dependent on antioxidant activity and reducing the intracellular calcium overload. These effects may protect cardiocytes by attenuating damage to mitochondrial function.

Mitochondrial activity is required for efficient function of the cardiovascular system. In response to cardiovascular injury, mitochondrial dysfunction may result in apoptosis and necrosis (21). A change in mitochondrial permeability is considered the primary event in the cell apoptosis cascade (22-24). The fluctuations in MMP result in cytochrome $c$ release, which can initiate caspase- 9 and -3 activation, and subsequent apoptosis (25). In the present study, AsIV was observed to protect against cell death by modulating mitochondrial membrane permeability, which may attenuate the release of cytochrome $c$ and reduce cardiocyte apoptosis. MitoK ${ }_{\text {ATP }}$ channels are located in the mitochondrial inner membrane, and the opening of these channels has been suggested to be protective against excessive mitochondrial $\mathrm{Ca}^{2+}$ accumulation, as mitoK $\mathrm{ATP}_{\text {ATP }}$ opening leads to depolarization, which reduces the driving force responsible for mitochondrial $\mathrm{Ca}^{2+}$ uptake (26). MitoK ${ }_{\text {АтP }}$ opening also regulates ROS generation; in early ischemia (30 min), mitoK ${ }_{\text {ATP }}$ opening can increase the production of ROS to begin cardiocyte protection by preconditioning. However, during late ischemia ( $24 \mathrm{~h})$ or reperfusion, mitoK $_{\text {ATP }}$ opening may reduce the production of ROS to reduce injury (27). Previous studies have indicated that mito$\mathrm{K}_{\mathrm{ATP}}$ serves an important function in the protection against ischemia-reperfusion injury by the mitoK ${ }_{\text {ATP }}$ opening agent, diazoxide, using an siRNA method and mitoK $_{\text {ATP }}$ blocker 5-HD $(28,29)$. Wakiyama et al (8) confirmed that mitoK ${ }_{\text {ATP }}$ opening may attenuate the release of cytochrome $c$, inhibit caspase-3 release, stabilize the MMP and inhibit apoptosis. Soeding et al (30) also demonstrated that levosimendan, a calcium sensitizer, preserves the contractile responsiveness of hypoxic human myocardium via mitoK ${ }_{\mathrm{ATPs}}$ and potential pERK1/2 activation.

To investigate the link between AsIV and the mitoK $_{\text {ATP }}$, the present study examined the effect of 5-HD on the effects of AsIV. It was observed that pretreatment with $50 \mu \mathrm{mol} / 15-\mathrm{HD}$, (a specific mitoK $\mathrm{ATP}_{\text {AT }}$ blocker) partially abrogated the protective effect of AsIV in the cardiocytes. 5-HD led to a reduction in viability and MMP, an increase in the levels of LDH and apoptosis and an increase in calcium overload, compared with the AsIV group. These data indicate that AsIV may protect cardiocytes from $\mathrm{H}_{2} \mathrm{O}_{2}$ injury through activating mitoK $\mathrm{K}_{\text {ATP. }}$. This concentration of 5-HD had no effect on cardiocytes alone (data not shown). It was also observed that AsIV produced a similar protective effect to the drug nicorandil, a mitoK $\mathrm{ATP}_{\mathrm{ATP}}$ activator.

Based on these findings, it was concluded that the opening of mitoK ${ }_{\text {ATPs }}$ may be the principal mechanism through which AsIV protects cardiocytes from $\mathrm{H}_{2} \mathrm{O}_{2}$ injury. AsIV may inhibit apoptosis in cardiocytes via several mechanisms: i) AsIV inhibits the initiation of apoptosis by reducing the formation 
of ROS and intracellular calcium overload; ii) AsIV inhibits the opening of MPTPs, which may reduce the release of apoptosis-inducing proteins, including cytochrome $c$, Smac and AIF; and iii) mitoK $_{\text {ATP }}$ opening may facilitate the signal transduction required for protection by AsIV. Further studies are required to elucidate whether other trigger factors underlie the protective effects of AsIV. Additionally, the detailed downstream mediators of mitoK ${ }_{\text {ATP }}$ require clarification.

\section{Acknowledgements}

This study was supported by the Science and Technology Department of Jilin Province (grant no. YYZX201260).

\section{References}

1. Murphy E and Steenbergen C: Mechanisms underlying acute protection from cardiac ischemia-reperfusion injury. Physiol Rev 88: 581-609, 2008.

2. Paradies G, Petrosillo G, Pistolese M, Di Venosa N, Federici A and Ruggiero FM: Decrease in mitochondrial complex I activity in ischemic/reperfused rat heart: involvement of reactive oxygen species and cardiolipin. Circ Res 94: 53-59, 2004.

3. Lemasters JJ, Theruvath TP, Zhong Z and Nieminen AL: Mitochondrial calcium and the permeability transition in cell death. Biochim Biophys Acta 1787: 1395-1401, 2009.

4. Baines CP: The mitochondrial permeability transition pore and ischemia-reperfusion injury. Basic Res Cardiol 104: 181-188, 2009.

5. Weiss JN, Korge P, Honda HM and Ping P: Role of the mitochondrial permeability transition in myocardial disease. Circ Res 93: 292-301, 2003.

6. Fryer RM, Eells JT, Hsu AK, Henry MM and Gross GJ: Ischemic preconditioning in rats: role of mitochondrial K(ATP) channel in preservation of mitochondrial function. Am J Physiol Heart Circ Physiol 278: H305-H312, 2000.

7. Garlid KD and Halestrap AP: The mitochondrial K(ATP) channel-fact or fiction? J Mol Cell Cardiol 52: 578-583, 2012.

8. Wakiyama H, Cowan DB, Toyoda Y, Federman M,Levitsky S and McCully JD: Selective opening of mitochondrial ATP-sensitive potassium channels during surgically induced myocardial ischemia decreases necrosis and apoptosis. Eur J Cardiothorac Surg 21: 424-433, 2002

9. Zhou JY, Fan Y, Kong JL, Wu DZ and Hu ZB: Effects of components isolated from Astragalus membranaceus Bunge on cardiac function injured by myocardial ischemia reperfusion in rats. Zhongguo Zhong Yao Za Zhi 300-302, 2000 (In Chinese).

10. Xu YC, Lan SL and Chen JY: Effects of huangqi sijun decoction on thyroxin and cyclic nucleotide levels in rat models with spleen deficiency syndrome. Chin Drug Res Clin Pharm 18: 291-293, 2007.

11. Guan FY and Yu XX: The protective effect of Astragalus membranaceus injection preconditioning on experimental myocardial ischemia/reperfusion injury in rats. Chin J Geront 30: 3126-3129, 2010.

12. Yang J, Li J, Lu J, Zhang Y, Zhu Z and Wan H: Synergistic protective effect of astragaloside IV-tetramethylpyrazine against cerebral ischemic-reperfusion injury induced by transient focal ischemia. J Ethnopharmacol 140: 64-72, 2012.
13. Meng LQ, Tang JW, Wang Y, et al: Astragaloside IV synergizes with ferulic acid to inhibit renal tubulointerstitial fibrosis in rats with obstructive nephropathy. Br J Pharmacol 162: 1805-1818, 2011.

14. Xie W and Du L: Diabetes is an inflammatory disease: evidence from traditional Chinese medicines. Diabetes Obes Metab 13: 289-301, 2011.

15. Qiu LH, Xie XJ and Zhang BQ: Astragaloside IV improves homocysteine-induced acute phase endothelial dysfunction via antioxidation. Biol Pharm Bull 33: 641-646, 2010.

16. Zhao Z, Wang W, Wang F, et al: Effects of Astragaloside IV on heart failure in rats. Chin Med 4: 6, 2009.

17. Guan FY, Li H, Sun W and Yang SJ: Protective effects of Astragaloside IV on hydrogen peroxide-induced injury of cultured neonatal rat myocardial cells. J Jilin Uni Med Ed 33: 211-214, 2007.

18. Zhang WD, Chen H, Zhang C, Liu RH, Li HL and Chen HZ: Astragaloside IV from Astragalus membranaceus shows cardioprotection during myocardial ischemia in vivo and in vitro. Planta Med 72: 4-8, 2006.

19. Xu XL, Chen XJ, Ji H, et al: Astragaloside IV improved intracellular calcium handling in hypoxia-reoxygenated cardiocytes via the sarcoplasmic reticulum Ca-ATPase. Pharmacology 81: 325-332, 2008

20. Yu WP, Xu GL, Shen CX and Qian ZY: Effects of crocetin on the apoptosis and the changes of its related regulating proteins caspase- 3 and $\mathrm{Bcl}-2$ induced by $\mathrm{H}_{2} \mathrm{O}_{2}$ in myocardial cell. Chin J Pathoph 22: 54-57, 2006

21. Smith MA and Schnellmann RG: Calpains, mitochondria and apoptosis. Cardiovasc Res 96: 32-37, 2012.

22. Grivicich I,Regner A,da Rocha AB, et al: Irinotecan/5-fluorouracil combination induces alterations in mitochondrial membrane potential and caspases on colon cancer cell lines. Oncol Res 15: 385-392, 2005

23. Kallio A, Zheng A, Dahllund J, Heiskanen KM and Harkonen P: Role of mitochondria in tamoxifen-induced rapid death of MCF-7 breast cancer cells. Apoptosis 10: 1395-1410, 2005.

24. Shrivastava A, Tiwari M, Sinha RA, et al: Molecular iodine induces caspase-independent apoptosis in human breast carcinoma cells involving the mitochondria-mediated pathway. J Biol Chem 281: 19762-19771, 2006.

25. Shi LG, Zhang GP and Jin HM: Inhibition of microvascular endothelial cell apoptosis by angiopoietin-1 and the involvement of cytochrome C. Chin Med J (Engl) 119: 725-730, 2006.

26. Murata M, Akao M, O'Rourke B and Marban E: Mitochondrial ATP-sensitive potassium channels attenuate matrix $\mathrm{Ca}(2+)$ overload during simulated ischemia and reperfusion: possible mechanism of cardioprotection. Circ Res 89: 891-898, 2001.

27. Shahid M, Tauseef M, Sharma KK and Fahim M: Brief femoral artery ischaemia provides protection against myocardial ischaemia-reperfusion injury in rats: the possible mechanisms. Exp Physiol 93: 954-968, 2008.

28. Wu Q, Bie P, Tang C and Zhang YJ: An experiment study on the role of bcl-2 in attenuation ischemia-reperfusion injury in liver graft in mice induced by mitoKATP channel opener diazoxide. J Clin Med Practi 12: 35-40, 2008.

29. Tratsiakovich Y, Gonon AT, Krook A, et al: Arginase inhibition reduces infarct size via nitric oxide, protein kinase $\mathrm{C}$ epsilon and mitochondrial ATP-dependent $\mathrm{K}^{+}$channels. Eur J Pharmacol 712: 16-21, 2013

30. Soeding PF, Crack PJ, Wright CE, Angus JA and Royse CF: Levosimendan preserves the contractile responsiveness of hypoxic human myocardium via mitochondrial K(ATP) channel and potential pERK 1/2 activation. Eur J Pharmacol 655: 59-66, 2011. 